Cahiers de la recherche sur les droits

Cahiers

Fur les Droits fondamentaux

6 | 2008

Pouvoirs exceptionnels et droits fondamentaux

\title{
Le modèle anglais
}

Jean-Claude Paye

\section{OpenEdition}

Journals

Édition électronique

URL : https://journals.openedition.org/crdf/6837

DOI : $10.4000 /$ crdf.6837

ISSN : 2264-1246

Éditeur

Presses universitaires de Caen

Édition imprimée

Date de publication : 31 décembre 2008

Pagination : 71-80

ISBN : 978-2-84133-259-5

ISSN : 1634-8842

Référence électronique

Jean-Claude Paye, «Le modèle anglais », Cahiers de la recherche sur les droits fondamentaux [En ligne], 6 | 2008, mis en ligne le 11 décembre 2020, consulté le 14 novembre 2022. URL : http:// journals.openedition.org/crdf/6837 ; DOI : https://doi.org/10.4000/crdf.6837 


\title{
Le modèle anglais
}

\author{
Jean-Claude PAYE
}

Sociologue

I. L'Anti-terrorism, Crime and Security Act 2001
A. Une suspension du droit
B. Une suspension du droit remise en cause par la Cour des Lords
C. État d'exception

II. The Prevention of Terrorism Bill
A. La fin de l'habeas corpus
B. Un droit subjectif
C. Une évaluation des services secrets

III. La fin de l'habeas corpus
A. État d'exception ou dictature
B. Un changement de régime

\section{Légalisation de la torture}

\section{The Terrorism Bill 2006}
A. De nouvelles incriminations
B. Un délit " d'insouciance "
C. Criminaliser un " climat " favorable au terrorisme
D. Des délits politiques
E. Une déterritorialisation du droit pénal

La décision-cadre de l'Union européenne est fortement inspirée de la loi anglaise, le Terrorism Act 2000, entré en application en février 2001. Cette loi définit le terrorisme comme une action ou une menace d'action qui «vise à influencer le gouvernement ou à intimider tout ou une partie de la population » ou comme «l'action ou la menace d'action qui a pour objectif de promouvoir une cause politique, religieuse ou idéologique ${ }^{1}$. Cet élément subjectif spécifie le délit comme terroriste quand celui-ci comprend
« une violence grave contre une personne» ou «la mise en danger de la vie d'une personne» ou « de graves dommages contre la propriété » ou "qui entraîne un risque sérieux pour la santé ou la sécurité de tout ou partie de la population » ou « qui interfère sérieusement ou qui perturbe sérieusement un système électronique».

C'est le but poursuivi qui définit l'acte comme terroriste. Le caractère subjectif de l'incrimination apparaît aussi dans la détermination des actes. Les termes « graves »

1. Terrorism Act 200o, http://www.uk-legislation.hmso.gov.uk/acts/acts200o/200ooo11.htm. 
et «sérieux» permettent une marge d'interprétation indéterminée. C'est le pouvoir qui désignera ou non les faits comme constituant une menace terroriste.

Par rapport aux lois antiterroristes antérieures, au nombre de huit de 1973 à 1996, le Terrorism Act 2000 s'inscrit dans la modernité. Il innove sur plusieurs points : le caractère subjectif des infractions, la portée générale de la loi ainsi que son utilisation dans le contrôle du Net. Cette législation doit donc se lire en parallèle avec la Regulation of Investory Power Bill (RIP Act). Cette dernière, adoptée en juillet 2000, fait de la simple intrusion dans un système informatique un acte terroriste.

Par rapport à son modèle anglo-saxon, la décisioncadre de l'Union européenne complète celui-ci en criminalisant des actes pouvant être accomplis lors des mouvements collectifs de protestations.

\section{L'Anti-terrorism, Crime and Security Act 2001}

Les attentats du 11 septembre ont permis au gouvernement britannique de faire adopter, en urgence, une nouvelle loi antiterroriste, L'Anti-terrorism, Crime and Security Act, qui fut promulguée le 14 décembre 2001. Par rapport au texte précédent, qui est toujours en application, cette nouvelle législation autorise, comme aux États-Unis, la détention illimitée, sans inculpation, d'un étranger suspect d'activités terroristes. L'article 21 permet de l'incarcérer indéfiniment grâce à un certificat émis par le ministre de l'Intérieur. Cet acte est établi sur base « d'une conviction raisonnable » que la présence d'une personne sur le territoire du Royaume-Uni représente « un risque » pour la sécurité nationale et qu'il y a « une suspicion raisonnable» qu'elle soit un terroriste international ${ }^{2}$. Aucun élément objectif n'est nécessaire pour justifier la décision. Le terme de «conviction» est entièrement subjectif.

Est terroriste « une personne qui est concernée par le fait de commettre, de préparer ou d'étudier des actes de terrorisme international» ou «qui est un membre appartenant à un groupe terroriste international » ou qui «a des liens avec un tel groupe ». La notion de «lien» n'est aucunement précisée. Elle est encore plus indéfinie et extensible que l'approche développée par le Terrorism Act 2000, qui poursuivait toute personne qui « supporte ou assiste» un tel groupe. Cela permet une extension incontrôlable des possibilités de poursuites, qui pourraient, par exemple, concerner un comité de défense de personnes incarcérées dans le cadre de cette loi.

Le certificat émis par le ministre de l'Intérieur permet la détention de la personne incriminée dans les termes de l'Immigration Act de 1971, c'est-à-dire «à des fins d'expulsion ou de retour dans le pays d'origine». La possibilité de détention, pour une période indéfinie, s'impose quand l'expulsion est empêchée par un accord international ou toute autre raison. Dans ce cas, la personne a le choix entre rester détenue indéfiniment au Royaume-Uni ou rentrer dans son pays d'origine et subir, là-bas, un ensemble de procédures dérogatoires aux normes juridiques internationales.

Le plus souvent, les pays d'origine des personnes incarcérées sont bien connus pour leurs violations systématiques des droits de l'homme. C'est d'ailleurs en ces termes que le débat fut présenté au Parlement ${ }^{3}$. L'étranger suspecté de terrorisme par le gouvernement anglais entre ainsi dans un système global de non-droit, dont toutes les issues lui sont fermées.

\section{A. Une suspension du droit}

En permettant une détention potentiellement illimitée de ces personnes, cette loi opère une suspension du droit pour toutes les personnes n'ayant pas la citoyenneté britannique ou un droit de résidence ou ne bénéficiant pas d'un droit de protection en tant que réfugiées. Ainsi, la suspension de l'habeas corpus est moins étendue qu'aux États-Unis, où tous les étrangers sont concernés.

Afin de faire voter cette loi, le gouvernement britannique a institué une dérogation à la Convention européenne des droits de l'homme. Elle se base sur la notion d'état d'urgence et fait ainsi exception à l'article 5 de cette convention qui garantit la liberté des personnes.

Selon l'article 15 de cette convention, les mesures dérogatoires doivent se limiter au minimum strictement nécessaire requis par la situation. Ce qui, comme l'a estimé la Special Immigration Appeal Commission (SIAC), n'est pas rencontré par la mesure de détention illimitée. Cette cour spéciale a été créée afin d'assurer un contrôle sur les détentions. La loi ne permet de faire appel qu'auprès de cette commission. Cela n'a pas empêché celle-ci d'ordonner la libération de neuf personnes sur les onze détenues dans le cadre de cette loi, les deux autres ayant choisi l'expulsion. Le gouvernement a réagi au jugement en annonçant son intention de recourir à d'autres procédures lui permettant de poursuivre l'incarcération. Il a ainsi clairement annoncé qu'il n'était pas prêt à respecter le jugement d'une cour spéciale qu'il a lui-même installée. Il a fait appel et la cour de deuxième instance lui a donné raison sur le fond, sur la question de la discrimination entre ressortissants étrangers et citoyens britanniques ${ }^{4}$.

\section{B. Une suspension du droit remise en cause par la Cour des Lords}

Le 22 décembre 2004, le secrétaire général du Conseil de l'Europe a demandé l'abrogation immédiate du Terrorism Act 2001: «La législation antiterroriste dans le Royaume-Uni doit être changée de manière urgente. Nous ne gagnerons pas la guerre contre le terrorisme si nous 
minons les fondations de nos sociétés démocratiques $»^{5}$. Cette prise de position fait suite à un jugement émis, le 16 décembre 2004, par la Cour d'appel de la Chambre des Lords, la plus haute instance judiciaire britannique, considérant comme illégale et contraire à la Convention européenne des droits de l'homme la détention illimitée, sans inculpation et sans procès, d'étrangers soupçonnés d'activités terroristes ${ }^{6}$.

Le jugement résulte d'une saisie opérée par 9 détenus, ceux-là mêmes qui avaient obtenu une première victoire, en juillet 2002, devant la Commission spéciale d'appel des affaires d'immigration (SIAC) et qui, cependant, avaient vu leur demande de libération rejetée, une nouvelle fois, par la Cour d'appel de Londres, en août 2004.

Aucun des requérants n'est l'objet de poursuites judiciaires, ni incriminé d'aucune charge. Les appelants contestent la légalité de leur détention, en affirmant qu'elle est en contradiction avec les obligations prises par le RoyaumeUni vis-à-vis de la Convention européenne des droits de l'homme et, ainsi, avec l'Human Rights Act 1998, qui intègre cette convention dans le droit britannique.

Par cet avis, la Cour a donné raison aux requérants. Le jugement reconnaît bien que l'Immigration Act 1971 offre la possibilité de détenir un individu ne disposant pas de la nationalité britannique en vue de procéder à son extradition, mais précise que cette loi n'accorde aucun mandat permettant une détention longue ou indéfinie.

\section{C. État d'exception}

À travers les attendus du jugement, apparaît clairement l'opposition entre une définition classique de l'état d'exception, limité dans le temps et objectivement défini, défendue par la Cour, et celle du gouvernement, voulant installer une suspension indéfinie et incontrôlable des libertés constitutionnelles.

Le jugement stipule que ce type d'incarcération, illimitée et sans jugement, autorisée par la nouvelle loi antiterroriste, le Terrorism Act 2001, est bien en contradiction avec l'article 5 de la Convention européenne des droits de l'homme, qui garantit la liberté des personnes. Le jugement invalide, par là même, la dérogation établie par le gouvernement à cet article de la Convention. Cette dérogation fait référence à l'article 15 de ce texte qui permet, « en temps de guerre ou d'autre urgence publique menaçant la vie de la nation », de déroger au principe de la liberté individuelle.

La Cour rejoint l'argument présenté par les requérants selon lequel les dérogations à l'article 5 , étant étroitement circonscrites aux situations de guerre ou d'urgence mettant en péril la vie de la nation, ne sont pas applicables à la situation présente.
Pour la Cour, l'état d'exception est limité dans le temps. Il doit faire face à un danger imminent ou à des circonstances exceptionnelles qui doivent être objectivement déterminées.

La Cour s'oppose à l'argumentation présentée par l'Attorney General (ministre de la Justice), pour qui une situation d'urgence n'est pas nécessairement temporaire et peut couvrir un nombre considérable d'années ${ }^{7}$. Pour lui, il est du ressort du pouvoir exécutif de protéger les populations. Il se doit donc de résister « à l'imposition de limites temporelles artificielles » aux procédures d'exception.

Pour le pouvoir exécutif, la question de la suspension des libertés est «d'ordre purement politique», dans le sens étroit du terme, c'est-à-dire qu'elle relève de sa propre initiative et du contrôle du Parlement. Comme «c'est la fonction du politique, et non celle d'instances judiciaires, de résoudre des questions politiques ${ }^{8}$, la question de la dérogation au droit doit échapper aux différentes instances judiciaires.

À cette position, le tribunal oppose la conception selon laquelle le rôle des cours consiste dans le contrôle de la légalité des actes des différents pouvoirs. Ce faisant, la plus haute instance judiciaire britannique rappelle le principe de la séparation des pouvoirs.

\section{The Prevention of Terrorism Bill}

Le jugement est un simple avis sans force contraignante. Le gouvernement peut ne pas en tenir compte. Mais celui-ci a finalement considéré que la prise en considération du jugement était une bonne occasion de légitimer la généralisation de dispositions d'exception à l'ensemble de la population.

The Prevention of Terrorisme Act 2005 se présente comme non discriminatoire, puisqu'il concerne tout autant les citoyens britanniques que les étrangers.

Ainsi, ce que G.W. Bush n'a pas réussi à imposer aux États-Unis, la possibilité pour le gouvernement de prendre, dans le cadre de la lutte antiterroriste, des mesures remettant en cause le droit des citoyens de disposer d'euxmêmes, Tony Blair est parvenu à l'imposer au RoyaumeUni.

\section{A. La fin de l'habeas corpus}

La nouvelle loi anglaise, The Prevention of Terrorism Bill $^{9}$, finalement votée le 11 mars 2005, autorise le ministre de l'Intérieur à prendre des mesures de contrôle pouvant conduire aux arrêts domiciliaires d'une personne, lorsqu’il «a des raisons fondées de soupçonner qu’un individu

5. Statewatch News Online, 22 décembre 2004, http://www.statewatch.org/news/archive2004.htm.

6. Opinions of the Lords of Appeal for judgement in the cause A (FC) and others (FC) (Appellants) v. Secretary of State for the Home Department (Respondent), House of Lords, session 2004-05 [2004], UKHL 56, 16 décembre 2004, http://www.statewatch.org/news/2004/dec/belmarsh-appeal.pdf.

7. Ibid., p. 15-16.

8. Ibid., p. 17 .

9. Prevention of Terrorism Bill, http://www.homeoffice.gov.uk/docs4/terrorism_bill.pdf. 
est ou a été impliqué dans une action liée au terrorisme ». Il pourra également lui interdire l'utilisation d'un téléphone mobile, limiter son accès au Net, l'empêcher d'avoir des contacts avec certaines personnes, l'obliger à être chez lui à certaines heures, autoriser la police et les services spéciaux à avoir accès à toute heure à son domicile. Il a également la possibilité de limiter son accès à un emploi ou à une occupation.

La liste de quinze mesures de contrôle, prévues par la loi, n'est pas exhaustive. L'énumération est établie à titre d'exemple. Le gouvernement a la possibilité d'introduire indéfiniment de nouvelles dispositions de limitation de la liberté de mouvement des personnes concernées. Si cellesci ne respectent pas ces ordres, ils sont passibles d'emprisonnement.

Ces dispositions peuvent être prises lorsque le ministre de l'Intérieur considère que l'individu présente un danger pour la sécurité nationale, mais que les éléments en sa possession ne lui permettent pas de porter l'affaire devant un tribunal. Le ministre de l'Intérieur a déclaré que les mesures de contrôle peuvent être prises sur «base d'un avis fondé donné par les services de sécurité qu'il y a une suspicion raisonnable qu'un individu est ou a été concerné par le terrorisme ${ }^{10}$.

\section{B. Un droit subjectif}

Ce qui justifie la décision de placer une personne sous contrôle ne se trouve pas dans des éléments objectifs, mais dans le soupçon qui porte sur elle ou dans l'intention qui lui est attribuée. L'activité terroriste est définie comme « la commission, la préparation ou l'instigation d'actes de terrorisme », mais aussi « quand elle est conduite pour donner aide ou assistance à des individus dont on sait ou on pense qu'ils sont impliqués dans des activités ayant rapport au terrorisme » ou quand elle a pour but «d'encourager la commission, la préparation ou l'instigation de tels actes » ou d'encourager celui « qui a l'intention de le faire ${ }^{11}$

Ainsi la loi ne porte pas sur des actes définis, mais punit l'aide à des personnes qui sont simplement soupçonnées d'actions ou d'intentions liées au terrorisme. Cette notion est particulièrement indéterminée et subjective. Son champ d'application est très large, quasiment illimité et totalement incontrôlable. Qu'est-ce qu'une activité liée au terrorisme? Est-ce, par exemple, avoir hébergé des personnes, qui plus tard seront soupçonnées de participer ou d'avoir eu l'intention de participer à des actions terroristes désignées comme terroristes ou faire partie d'un comité de soutien de prisonniers politiques?

La réaction de la Chambre des Lords fut problématique pour le gouvernement. Elle a d'abord rejeté le projet en considérant le texte comme une atteinte aux libertés. Elle finit par l'accepter après trente heures de débat et après avoir obtenu que les mesures de contrôle soient pri- ses avec l'aval d'un tribunal. En vertu du nouveau texte, le ministre de l'Intérieur devra s'adresser à un juge pour décider d'une assignation à résidence, mais, en cas d'urgence, il pourra immédiatement ordonner des mesures moindres et demander le feu vert d'un tribunal dans les sept jours. Surtout, la Chambre haute a imposé que le vote de la loi soit assorti d'une clause (Sunset clause) de révision d'un an.

Si la décision est prise par le ministre de l'intérieur en accord avec un tribunal, cette garantie judiciaire, obtenue par la Chambre des Lords, n'a rien à voir avec une procédure judiciaire classique qui garantit les droits de la défense. Ici, la défense n'a pas accès au dossier comprenant les éléments à charge et n'a aucune possibilité de contester ces éléments. Seuls ont accès à ces données le juge et des « avocats spéciaux » désignés par le ministre de l'Intérieur. Ces derniers sont chargés de transmettre le point de vue de la défense, sans fournir à celle-ci les éléments de « preuve » retenus contre elle et sans lui donner la possibilité de les contredire. La décision est prise en l'absence de la personne incriminée.

Contrairement à ce qu'affirme le ministre de l'Intérieur, cette procédure d'exception n'a rien à voir avec la détention préventive de longue durée, telle qu'elle est appliquée en matière de terrorisme, par des pays comme l'Espagne ou l'Allemagne. Dans ce cadre, la détention est uniquement ordonnée par un juge, la personne incriminée peut connaître les charges qui sont retenues contre elle. La défense a la possibilité de contester les preuves ou les raisons de la détention.

À travers la procédure anglaise, on assiste à une suppression de la présomption d'innocence qui s'applique aux personnes poursuivies dans un cadre judiciaire.

\section{Une évaluation des services secrets}

Les mesures de contrôle peuvent être prises sur la base de renseignements fournis par un service de sécurité. Cette source peut être étrangère au Royaume-Uni. Lors des débats au sein de la Chambre des Lords, le gouvernement avait concédé que l'accusation ne pourrait pas utiliser des éléments à charge obtenus par le biais de la torture. Cependant, le gouvernement ne semble pas avoir renoncé à utiliser de tels renseignements. The Independant a relaté les déclarations du ministre des Affaires étrangères affirmant que si «la torture est complètement inacceptable, le pays ne peut pas ignorer les renseignements obtenus par ce biais par les Américains, surtout si la vie de 3000 personnes est en jeu» ${ }^{12}$.

L'évaluation faite par les services de sécurité, devant servir de base à la décision du ministre de l'Intérieur, peut s'appuyer sur une grande diversité de sources, dont nombre d'entre elles ne pourraient pas servir de preuve dans une procédure judiciaire. Il ne s'agit pas seulement d'interception de communications téléphoniques ou des e-mails 
mais de l'ensemble des procédures intrusives mises en œuvre par un service secret, telles la vidéo surveillance, l'infiltration, les informations obtenues par des agents sous couverture... Les renseignements contenus dans les rapports ou exposés effectués par ce type d'agent ou par des informateurs agents sont évalués selon une échelle de vraisemblance avec «des degrés variés de véracité et de précision ».

L'évaluation effectuée par les services de renseignement sur le caractère dangereux pour la sécurité nationale d'un individu se détache de la constatation d'un acte déterminé. Un individu peut être considéré comme dangereux et faire l'objet de mesures de contrôle s'il est soupçonné d'être « membre ou associé à des organisations suspectes ».

Il n'est pas nécessaire que les «preuves » établies sur la base des renseignements fournis par les services secrets soient objectives. Elles peuvent se réduire à l'évaluation subjective d'un danger potentiel auquel on attribue un niveau déterminé de dangerosité.

Les notes exploratoires de la loi insistent sur le caractère préventif des dispositions de contrôle ${ }^{13}$, ces mesures devant prévenir des attentats terroristes en Grande-Bretagne.

Le sort réservé par le gouvernement aux prisonniers de nationalité anglaise incarcérés à Guantanamo est un bon exemple d'application anticipée de cette loi. Quatre Britanniques, détenus à Guantanamo depuis plus de trois ans, ont été libérés en janvier 2005. Après avoir été interrogés par la police antiterroriste anglaise, ils furent libérés un jour plus tard. Aucune charge ne fut retenue contre eux. Ce fait n'empêche pas le ministre de l'Intérieur de les considérer comme présentant un danger terroriste pour la Grande-Bretagne. Il a pris, à leur égard, des mesures de contrôle, telles que l'interdiction de voyager à l'étranger et l'impossibilité d'obtenir un passeport. Il justifia ces mesures sur la base de renseignements obtenus lors d'interrogatoires à Guantanamo ${ }^{14}$. Ainsi, sur base de « renseignements » donnés par les États-Unis, obtenus dans des conditions de torture ou de mauvais traitements, et sans possibilité de vérification ou de contestation, les prisonniers libérés de Guantanamo demeurent dans un système international de non-droit.

\section{La fin de l'habeas corpus}

L'élément le plus significatif de cette nouvelle loi anglaise, The Prevention of Terrorism Bill, est le fait qu'elle généralise la suspension du droit aux nationaux. Elle met fin à un double système d'organisation juridique : État de droit pour les nationaux et violence pure pour les étrangers. La suppression de l'habeas corpus est généralisée à l'ensemble des habitants. On entre dans un état d'exception généralisé.

\section{A. État d'exception ou dictature}

En imposant une clause de révision au Prevention Security Act 2005, en permettant que la loi soit évaluée après un an, la Chambre des Lords maintient celle-ci dans le cadre formel d'un état d'exception, puisque ces mesures pourront être abrogées à cette date. Le gouvernement ne voulait fixer aucune limite temporelle, la lutte antiterroriste étant conçue comme une guerre de longue durée contre un ennemi multiforme. Il n'a cependant aucunement renoncé à son projet et veut profiter de la procédure de révision pour faire adopter les mesures de contrôle libérées de leurs contraintes temporelles.

Cependant, cette loi ne s'inscrit plus que formellement dans un état d'exception. Elle donne au ministre de l'Intérieur des pouvoirs de magistrat. Une personne est désignée comme terroriste non par un jugement, mais par un certificat établi par un représentant du pouvoir exécutif. Ce dernier ne doit, à aucun moment, justifier une décision qui s'applique à de simples suspects. Les éléments objectifs, devant servir de base à ces soupçons, ne sont même pas nécessaires puisqu'ils restent secrets. Il suffit que l'autorité administrative affirme les détenir et que cette déclaration soit corroborée par un tribunal. Quelle est la garantie d'un contrôle judiciaire qui s'exerce sans possibilité pour la défense de faire valoir des droits, même celui de connaître ce qu'il lui est reproché? Quelle indépendance le pouvoir judiciaire peut-il faire valoir dans un processus de décision dans lequel il n'a pas les moyens de vérifier les informations qui lui sont données ainsi que les moyens de preuve? De plus, cette mesure établit un lien explicite entre les modes de gestion administrative de l'immigration et l'organisation de la lutte contre le terrorisme. Elle fait de celle-ci un outil de la première.

Cette loi est un bon exemple d'un droit purement subjectif, permettant un maximum d'interprétation et d'un droit qui assure la primauté de la conviction sur les faits. Le pouvoir exécutif concentre entre ses mains l'ensemble des pouvoirs, dont les prérogatives judiciaires. Cette loi fait entrer le Royaume-Uni dans une nouvelle forme de régime politique que la théorie du droit désigne comme dictature.

L'Antiterrorism, Crime and Security Act 2001 ainsi que le Prevention of Terrorism Act 2005 représentent un pas supplémentaire dans le démantèlement de l'État de droit.

Ces lois doivent être envisagées comme la première étape d'un processus destiné à étendre les mesures de suspension du droit à l'ensemble de la population, nationaux compris. Ce projet a déjà été dévoilé par le ministre de l'Intérieur. Il a également parlé de la possibilité de faire juger secrètement de simples suspects par des juridictions spéciales. Le prévenu n'aurait pas le choix de son avocat. Celui-ci serait désigné par le pouvoir exécutif, sur base d'une liste agréée par les services secrets. 


\section{B. Un changement de régime}

Entre le Terrorism Act 2000 et ces nouvelles lois, il existe un changement qualitatif. La première législation représente le point ultime d'une mutation interne du droit pénal qui se réorganise autour de la procédure d'exception. Il s'agit d'un démantèlement de l'État de droit effectué dans le cadre de celui-ci. Dans l'Anti-terrorism, Crime and Security Act, l'exception, la capacité offerte au pouvoir exécutif de se saisir, sans contrainte juridique, d'une personne étrangère et, dans le Prevention of Terrorism 2005, de toute personne soupçonnée de terrorisme, est un premier élément constitutif d'un changement de régime politique, d'une mutation qui concerne l'ensemble de la population, qui engage celle-ci dans un état d'exception généralisé et dans un état d'urgence qui s'installe dans la durée. Ici, la dérogation s'avère être un acte de violence pure, extérieure au système juridique institué. Elle est l'élément à partir duquel se construit une nouvelle forme de gouvernement qui met fin à la séparation formelle des pouvoirs.

Comme l'a indiqué le ministre de l'Intérieur, David Blunkett, le gouvernement a pour objectif de généraliser la possibilité de se saisir de la personne des ressortissants britanniques accusés de terrorisme et de les incarcérer sans jugement pour une période indéterminée. Ainsi, l'exception est non seulement l'élément de transformation de l'ordre juridique existant, mais aussi la base sur laquelle se construit un nouvel ordre politique, et cela, aux niveaux national et mondial.

Les nécessités internationales sont d'ailleurs mises en avant par le gouvernement afin de justifier cette mesure. Le ministre de l'Intérieur a «considéré que la présence continuelle et libre au Royaume-Uni de personnes suspectées de terrorisme, ne pouvant être déplacées vers des pays tiers, avait des effets néfastes pour le pays dans la situation d'urgence actuelle. Le gouvernement au plus haut niveau en a conclu que cette situation aurait un impact négatif sur la capacité du Royaume-Uni à construire et à préserver une coalition internationale efficace dans la lutte contre le terrorisme ${ }^{15}$.

Comme aux États-Unis, l'exception est l'élément à partir duquel se construit un nouvel ordre juridique et politique au niveau international. Il faut considérer cette mesure en rapport avec le rôle que joue la Grande-Bretagne dans la mise en place d'une structure impériale sous direction américaine.

\section{Légalisation de la torture}

Le ministre de l'Intérieur (Home Secretary) et le Premier ministre Tony Blair veulent lever tout obstacle aux nouvelles législations. Ce dernier déclara, lors de la présentation du plan gouvernemental de lutte contre le terrorisme: "nous devons légiférer davantage, en incluant, si nécessaire, l'amendement de l'Human Rights Act, en respect avec l'interprétation de la Convention européenne des droits de l'homme » ${ }^{16}$. Michael Howard a également promis une révision de cet acte par un éventuel gouvernement conservateur.

L'Human Rights Act, qui est devenu une loi en 2000, oblige les juges à tenir compte de la jurisprudence de la Cour européenne des droits de l'homme dans leurs décisions. Charles Clarke a remis en cause la jurisprudence de cette cour sur la protection des réfugiés. Celle-ci interdit la torture, ainsi que les traitements inhumains et dégradants. Elle s'oppose au renvoi des réfugiés, lorsque ceuxci sont menacés de tels traitements. Cette jurisprudence est un obstacle au plan du gouvernement, qui veut renvoyer des étrangers, accusés d'apologie ou de soutien au terrorisme, vers leur pays d'origine ou bien les déporter vers des pays tiers, même s'ils risquent la torture.

Cependant, malgré sa reconnaissance de la Convention européenne, Londres a déjà engagé des négociations avec la Libye, l'Algérie et le Liban, afin de leur réexpédier ces «indésirables». Londres a signé, le 18 octobre 2005, un accord avec la Libye visant à lui transférer ses ressortissants, considérés en Angleterre "comme une menace pour la sécurité nationale». Pour cela, la Grande-Bretagne s'est contentée de l'engagement formel de ce pays, disant que ces prisonniers ne seraient pas maltraités à leur retour. Un premier accord avait déjà été signé avec la Jordanie et un autre est en préparation avec l'Algérie ${ }^{17}$.

Le gouvernement était conforté par un jugement, en août 2004, de la cour d'appel d'Angleterre et du pays de Galles, qui avait statué que des " preuves ", obtenues sous la torture dans des pays tiers, étaient recevables dans le cadre des procédures soumises à la commission spéciale des recours en matière d'immigration. Ne seraient pas valables les preuves obtenues directement par des agents britanniques ou avec leur participation, la torture restant interdite en Grande-Bretagne ${ }^{18}$. La Cour d'appel justifiait sa décision par le fait que la Convention européenne des droits de l'homme ne fait pas partie des lois britanniques. Ainsi, les tribunaux ne pourraient exclure de telles preuves ${ }^{19}$. Par une majorité $2 / 1$, la Cour d'appel avait statué que si les preuves sont obtenues par des agents d'un pays étranger, sans implication de la Grande-Bretagne, il n'y a pas lieu d'enquêter sur les origines de celles- $\mathrm{ci}^{20}$.

15. A, X, and Y and others v. Secretary of State for the Home Department (2002), EWCA Civ 1502, paragraphe 110.

16. M. Berlins, «Human rights in peril», The Guardian, 31 octobre 2005.

17. A. Thoraval, «Accord d'extradition entre Londres et Tripoli », Le Monde, 20 octobre 2005.

18. "Admettre des preuves arrachées sous la torture dans les tribunaux britanniques, c'est accepter l'inacceptable", Amnesty International, index AL:

EUR 45/ 044 / 2005, 17 octobre 2005, http://web.amnesty.org/library/index/fraEUR450442005.

19. C. Dyer, «Lords to rule on evidence from torture », The Guardian, 17 octobre 2005.

20. C. Dyer, «UK wins allies in challenge to torture ruling», The Guardian, 18 octobre 2005. 
Le 8 décembre 2005, la Cour d'appel de la Chambre des Lords a, dans un jugement unanime, remis en cause la décision de la Cour d'appel. Elle a décidé que les preuves obtenues sous la torture ne sont pas admissibles par les tribunaux anglais et particulièrement par le SIAC. Cette décision a plusieurs implications en ce qui concerne l'action du gouvernement, qui doit revoir tous les cas où de telles preuves ont été utilisées. Il doit notamment s'abstenir d'expulser des personnes vers des pays qui pratiquent la torture. Le gouvernement doit indiquer le lieu où les preuves ont été produites. S’il ne peut révéler ses sources pour des raisons de sécurité, il doit en produire d'autres. Le jugement oblige ainsi le ministre de l'Intérieur à revoir tous les cas de suspects dont les preuves les désignant comme terroristes ont été obtenues par des voies tenues secrètes. C'est le cas de la moitié des huit personnes détenues, sans que le gouvernement dispose de charges contre elles ${ }^{21}$.

Le jugement de la Cour d'appel de la Chambre des Lords impose au Special Immigration Appeals Commission (SIAC) d'enquêter afin de déterminer s'il y a une suspicion raisonnable permettant de penser que les preuves présentées ont été obtenues sous la torture. Trois juges estiment que, si il y a un doute sur le fait que la torture ait ou ait pu être utilisée, la preuve ne peut être admise ${ }^{22}$. Le jugement de la Cour implique que, au lieu que le gouvernement doive démonter qu'il n'y a aucune implication active des services britanniques dans le fait d'utiliser la torture pour obtenir des informations, il revient au procureur de prouver que ces méthodes impropres n'ont été, d'aucune façon, utilisées pour obtenir ces renseignements.

\section{The Terrorism Bill 2006}

Le 30 mars 2006, le projet de loi antiterroriste, déposé par le gouvernement britannique après les attentats du mois de juillet 2005, est entré en vigueur. Cette loi représente un nouveau pas en avant dans la capacité offerte au pouvoir exécutif de criminaliser, non seulement toute action politique, mais aussi toute parole d'opposition radicale ou de soutien à ces actions. La matérialité des faits n'est plus nécessaire pour poursuivre des comportements, ni l'intention attribuée aux personnes incriminées. Il s'agit là de l'apport spécifique de cette loi. Il suffit qu'une personne, n'importe laquelle, déclare se sentir incitée à commettre des actes terroristes, par des paroles qui ont été prononcées par un tiers, pour que l'on puisse poursuivre l'auteur du discours. La personne qui parle est donc responsable de la manière dont ses déclarations peuvent être reçues, quel que soit son objectif. Il n'est pas non plus nécessaire qu'il y ait un lien matériel entre le contenu du discours prononcé, par exemple entre des paroles de soutien à la résistance palestinienne et les actes qu'elles auraient
« incités », par exemple la pose de bombes dans le métro de Londres. Pour être poursuivi, il suffit qu'un tribunal estime que ces paroles ont créé un « climat» favorable au terrorisme.

Cette loi donne également une compétence universelle aux tribunaux britanniques, celle de nommer comme terroriste toute action politique, développée n'importe où dans le monde, et ainsi de criminaliser tout soutien, effectif ou par la parole, à ces actes.

\section{A. De nouvelles incriminations}

La loi innove vraiment avec la création de la notion d'encouragement au terrorisme qui «s'applique à une déclaration qui peut être comprise par un membre du public, auquel elle est destinée, comme un encouragement direct ou indirect, ou comme une autre incitation à commettre, préparer ou concevoir des actes de terrorisme ou des délits repris sous cette Convention ${ }^{23}$.

L'infraction existe, non seulement quand la personne veut pousser à l'action terroriste ou qu'elle sait qu'elle encourage le terrorisme par ses déclarations, mais aussi quand une "personne raisonnable » peut conclure que la déclaration semble l'inciter au terrorisme, et cela indépendamment de l'intention de l'orateur. Ainsi, l'encouragement indirect au terrorisme est défini comme une déclaration glorifiant la commission d'actes de terrorisme, déclaration à travers laquelle des personnes peuvent « raisonnablement conclure qu'elles ont été encouragées à commettre de tels actes ».

En résumé, la loi adopte une incrimination « d'incitation directe », quand la personne défend directement la violence, et «d'incitation indirecte», quand la personne qui parle ne défend pas directement la violence, mais que sa déclaration peut être entendue comme quelque chose susceptible d'y encourager.

\section{B. Un délit « d'insouciance »}

Les incriminations d'encouragement au terrorisme et d'incitation indirecte ne requièrent pas qu'il y ait intention de pousser d'autres personnes à commettre des actes criminels. Une personne peut commettre ces délits sans s'en rendre compte. Le délit d'incitation indirecte existe si une personne qui publie une déclaration est simplement « insouciant » quant à la possibilité que son discours soit ou non compris comme un encouragement au terrorisme. La loi a une approche très large de la notion d'insouciance, elle comprend «les cas où une personne peut être tenue comme insouciante inclut chaque situation où la personne ne peut pas raisonnablement avoir manqué d'avoir connaissance de cette possibilité ${ }^{24}$. L'utilisation du terme

21. D. Wes Rist, «UK law lords rule against use of violence», Jurist, 8 décembre 2005, http://jurist.law.pitt.edu/paperchase/2005_12_08_indexarch. php\#113404465219984764.

22. «Opinions of the Lords of Appeal for judgement in the cause A (FC) and others (FC) (Appellants) v. Secretary of State for the Home Department (Respondent) (2004), House of Lords, Session 2005-06 [2005] UKHL 71, 8 décembre 2005, http://www.publications.parliament.uk/pa/ld200506/ ldjudgmt/jdo51208/aand.pdf.

23. Terrorism Bill, clause 1(1) http://www.opsi.gov/uk/acts/acts2006/ukga_2006011_en_pdf.

24. Ibid., clause 1(3). 
« inclut » suggère que cet article s'applique au cas ou la personne qui parle n'a pas conscience des effets que peuvent avoir ses mots.

Le gouvernement justifie l'introduction du délit «d'incitation indirecte au terrorisme» en arguant qu'il s'agit là d'une ratification de la Convention du Conseil de l'Europe relative à la prévention du terrorisme ${ }^{25}$, signée en avril 2005.

La Convention requiert des États «qu'ils adoptent de telles mesures qui sont nécessaires pour incriminer, sous les lois nationales, la provocation publique à commettre un délit terroriste, quand elle est commise illégalement et intentionnellement ${ }^{26}$.

Cette Convention introduit bien des notions telles que l'incitation directe ou indirecte au terrorisme, cependant, le délit est commis quand une déclaration publique, « avec l'intention d'inciter à commettre un acte terroriste », «cause un danger» tel qu'un délit puisse être commis. Un lien doit pouvoir être établi entre l'affirmation désignée comme provocatrice et l'acte qui doit être prévenu ${ }^{27}$. La loi britannique, si elle s'inscrit dans le sillage de la Convention du Conseil de l'Europe, va nettement plus loin que son modèle, puisque, ici, pour établir le délit, aucun élément d'intentionnalité, ni l'existence d'un délit concret, incité par la déclaration, n'est nécessaire.

\section{Criminaliser un " climat " favorable au terrorisme}

Les députés ont réintroduit une disposition, qui avait été écartée en janvier par la Chambre des Lords : la création du délit de «glorification du terrorisme ». Les Lords avaient estimé que cette nouvelle incrimination constituait un danger pour la liberté d'expression. Afin de contourner ce vote, le gouvernement avait réintroduit la notion de glorification, en en faisant un élément constitutif du délit «d'incitation indirecte au terrorisme ». Cependant, faire de la glorification du terrorisme une infraction spécifique a toujours été un objectif clairement affiché par le pouvoir politique, jusqu'à en faire un point du programme électoral du parti travailliste.

L'acharnement du gouvernement à vouloir réintroduire à tout prix cette notion ne peut se comprendre facilement que si l'on observe le degré d'arbitraire que permet déjà l'incrimination «d'incitation indirecte », dont la glorification était devenue, suite au vote des Lords, un élément constitutif.

Selon le gouvernement, cette nouvelle incrimination vise à punir ceux qui « louent ou célèbrent » les actes de terrorisme. Le pouvoir prétend qu'il veut avant tout sanctionner les imams radicaux, présentés comme des «prêcheurs de haine ». L'opposition conservatrice et libérale a défendu l'idée selon laquelle l'incrimination d'encouragement indirect au terrorisme était largement suffisante. Au contraire, pour le ministre Charles Clarke, cette incrimination serait trop étroite car elle ne couvrirait pas les écrits ainsi que les sites Internet.

Le terme de glorification n'est pas défini. En réponse à cette constatation, le ministre de l'Intérieur a déclaré qu'il ne serait d'ailleurs pas nécessaire de le préciser, puisque «ceux qui cherchent à recruter pour le terrorisme savent ce que cela signifie ${ }^{28}$.

L'intérêt du gouvernement à introduire ce nouveau délit se comprend davantage si on constate que les députés ont également adopté un amendement, selon lequel la police n'a pas besoin de demander un mandat à un juge afin d'obliger un fournisseur d'accès Internet à effacer des sujets désignés comme glorifiant le terrorisme. Le caractère vague de la nouvelle loi permet de détacher l'incrimination de préparation d'actes terroristes de tout support matériel. Il est ainsi possible de criminaliser le fait de visiter un site web «jihadist». On peut ainsi qualifier cette incrimination «d'usage inapproprié de l'Internet ${ }^{29}$.

L'utilisation de l'incrimination autorise toutes les interprétations. Une personne peut être poursuivie pour glorification du terrorisme si « les circonstances et la manière dont est faite la déclaration publique (en relation avec son contenu) déterminent qu'il serait raisonnable, pour des personnes du public auquel elle est adressée, d'admettre que l'exposé exprime les vues de cette personne ou avait son adhésion ${ }^{30}$.

Selon le ministre de l'Intérieur C. Clarke, cette incrimination est nécessaire pour agir contre des organisations «qui essaient de promouvoir le terrorisme et de créer un climat dans lequel un jeune homme impressionnable pourrait penser que les attentats suicides seraient une activité noble et sacrée ${ }^{31}$. Dans un même registre, C. Clarke a aussi déclaré que cette incrimination se justifie car «les personnes qui glorifient le terrorisme favorisent la création d'un climat dans lequel le terrorisme est perçu comme quelque chose d'acceptable $»^{32}$.

La déclaration, désignée comme glorifiant le terrorisme, peut se situer dans le passé, dans le futur ou avoir simplement un caractère d'ordre général. Le délit de glorification du terrorisme peut ainsi être appliqué à des faits anciens, sans rapport concret avec leur conséquence attribuée : le « climat », «l'atmosphère » terroriste actuelle. La durée pendant laquelle la déclaration peut être poursuivie

25. Council of Europe Convention on the Prevention of Terrorism, mai 2005, http://conventions.coe.int/Treaty/EN/Treaties/Html/196.htm.

26. Ibid., article 5(2).

27. Human Rights Watch, «Briefing on the Terrorism Bill 2005», novembre 2005, http://hrw.org/backgrounder/eca/uk1105/uk1105.pdf.

28. A. Travis, P. Winters, «Extremist muslim groups to be banned», The Guardian, 16 février 2006.

29. "A briefing document on the governments anti-terrorism proposals », p. 4, Protect Our Rights, http://www.blink.org.uk/docs/protectourrightsbriefing.pdf.

30. «The Terrorism Bill», House of Lords, Reasearch paper 05/ 66, 20 octobre 2005, p. 15, http://parliament.uk/commons/lib/research/rpo5-066pdf.

31. «Mps back ban on glorifing terror », $B B C$ news, http://news.bbc.w.uk/1/Li/uk_politics / 4714578.stm.

32. B. Gardiner, «Britain's House of Commons votes to outlaw the glorification of terrorism », http://cnews.canoe.ca/CNEWS/World/WarOnTerrorism/ 2006/02/015/1444273-ap.html. 
est quasiment indéterminée. La loi prévoit un délai de vingt ans qui suit le fait incriminé « à moins que le ministre de l'Intérieur ait pris un arrêté spécifique qui permet de prendre en compte des évènements advenus en dehors de cette période ${ }^{33}$.

\section{Des délits politiques}

Le Premier ministre a déclaré que la « glorification » ou «la justification » du terrorisme sont les comportements qui peuvent conduire à une exclusion du territoire, à justifier la fermeture de mosquées ou l'utilisation de «mesures de contrôle». Le gouvernement britannique a précisé les « comportements inacceptables » qui pourront conduire à l'expulsion de l'étranger incriminé : notamment le fait de « fomenter, justifier ou glorifier la violence terroriste », de « chercher à provoquer des actes terroristes » ou de «fomenter la haine pouvant mener à des violences entre les différentes communautés au Royaume-Uni ». Ainsi, la nouvelle loi donne ainsi une base légale aux décisions administratives de bannir les groupes dont le gouvernement estime qu'ils ont un rapport avec le terrorisme.

Comme les associations de défense des libertés civiles, le maire de Londres, Ken Livingstone, n'est pas convaincu par les déclarations du ministre Charles Clarke affirmant que les intentions du gouvernement n'étaient pas «d'étouffer la liberté d'expression ou le débat légitime sur les religions ou autres thèmes ${ }^{34}$. Il a ainsi affirmé que ces nouvelles dispositions ne résistent pas au «test Nelson Mandela ». "Si le texte avait été en place il y a vingt-cinq ans en Grande-Bretagne, les partisans de Nelson Mandela auraient-ils été expulsés de notre pays car ils soutenaient la campagne d'attentats à la bombe contre le régime raciste d'apartheid en place en Afrique du Sud? », a-il déclaré. «Si oui, alors le Parlement devrait s'insurger contre cette mesure ${ }^{35}$, a-il ajouté.

Pour comprendre toute la portée des nouvelles incriminations, celles d'incitation indirecte et de glorification du terrorisme, on doit les relier au Terrorism Act 2000 qui définit comme terroriste l'acte qui entraîne des «dommages sérieux à la propriété», qui « a pour objectif d'influencer le gouvernement ou d'intimider la population ou une partie ce celle-ci » et qui « le fait avec le but de promouvoir une cause politique, religieuse ou idéologique ${ }^{36}$.

Une telle définition du terrorisme permet de criminaliser, non seulement des actions ayant des références religieuses, mais aussi des mouvements sociaux de défense des droits politiques et économiques. On voit tout de suite l'utilisation que le pouvoir peut faire de ces nouvelles incriminations pour s'attaquer à des actions non violentes, des paroles de solidarité vis-à-vis de personnes ayant commis des actes désignés comme terroristes ou simplement des déclarations, des prises de position hostiles à l'action politique, économique ou sociale du gouvernement ou d'une organisation internationale.

Par rapport au Terrorism Act 2000, le Terrorism Act 2006 représente un nouveau pas dans le démantèlement des libertés publiques. Le Terrorism Act 2000, comme les lois antiterroristes adoptées au niveau de l'Union européenne, permet de s'attaquer à toute action politique que le gouvernement juge indue. Il criminalise le but de l'auteur d'un délit, celui de faire pression sur un gouvernement ou sur une organisation internationale. Il met en place un délit d'intention.

Le Terrorism Act 2006 remet en cause la possibilité d'exprimer une opinion politique qui soit inacceptable pour le gouvernement, que ce soit au niveau des affaires intérieures ou au niveau de sa politique internationale. Il s'écarte encore davantage de la matérialité des faits et s'inscrit pleinement dans le virtuel, puisque l'intention de la personne poursuivie d'obtenir par son discours un résultat déterminé n'est même plus nécessaire, seule compte la manière dont peuvent être interprétées ses paroles, sans qu'il y ait nécessité d'un rapport objectif entre le discours prononcé et les faits incriminés.

\section{E. Une déterritorialisation du droit pénal}

Le Terrorism Bill 2006 représente un pas important par rapport au Terrorism Act 2000 dans la capacité de criminaliser des groupes de plus en plus importants de la population. La clause 21 de la nouvelle loi permet une extension des raisons qui permettent une mise hors la loi des personnes stigmatisées.

Le Terrorism Act 2000 permet déjà de poursuivre les individus et les organisations violentes. Être membre d'une telle organisation est un délit ${ }^{37}$ qui peut conduire à une peine de dix ans d'emprisonnement. Le soutien à de à de telles organisations est également poursuivi ${ }^{38}$. La notion de soutien ne se limite pas à ses aspects financiers ou matériels, il est suffisant « de supporter ou de favoriser les activités d'une organisation par n'importe quel moyen ». Est également poursuivi le fait «de porter un signe distinctif ou un vêtement qui peut conduire à une suspicion raisonnable que l'on est membre ou que l'on supporte une organisation interdite ${ }^{39}$.

La nouvelle loi permet, quant à elle, à travers la notion de glorification, de s'attaquer à des groupes non violents, de criminaliser des individus qui supportent des groupes violents, simplement par la parole ou par des écrits. Elle s'attaque à une glorification «illégale » ${ }^{40}$.

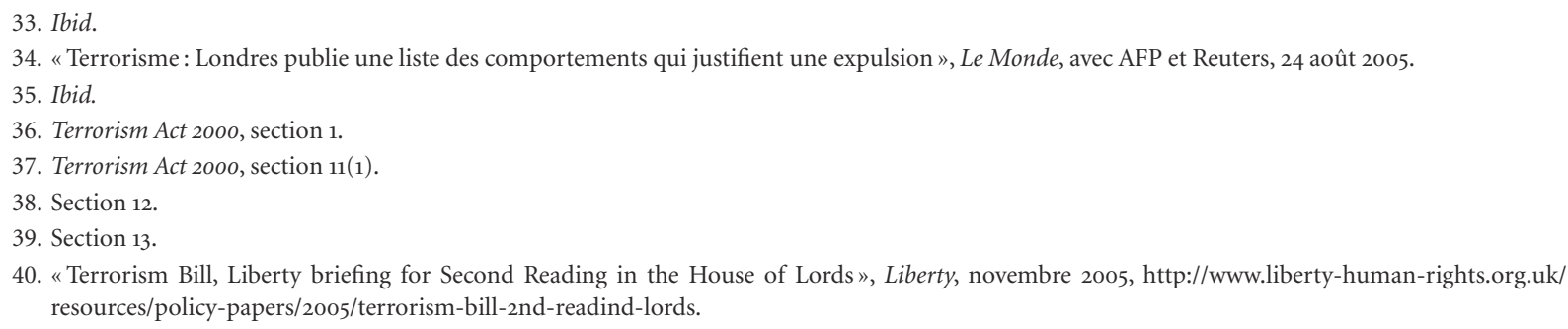


L'article 17 du Terrorism Bill prévoit une extension extraterritoriale de la compétence des tribunaux britanniques en ce qui concerne les nouveaux délits : l'encouragement au terrorisme, la dissémination de publications, la préparation et l'entraînement à des actes de terrorisme, ainsi que pour les infractions reprises dans le Terrorism Act 2000 : l'appartenance à une organisation interdite, la conspiration, la tentative, l'assistance et l'encouragement à commettre des actes terroristes. Quiconque commet un de ces actes à l'extérieur du Royaume-Uni peut être poursuivi par un tribunal britannique. Cette disposition ne concerne pas seulement les nationaux, mais toute personne impliquée, quelle que soit sa nationalité.

En fait, la nouvelle loi anglaise donne au gouvernement la possibilité de proscrire toute organisation politique, d'emprisonner ou de déporter toute personne qui exprime, a exprimé ou exprimera une opinion politique différente de celle du gouvernement concernant un conflit violent n'importe où dans le monde.

Par exemple, la déclaration du journaliste John Pilger, lors d'une interview à la télévision australienne ${ }^{41}$, selon laquelle la résistance face aux forces américaines en Irak est légitime et désirable, tomberait sous le champ d'application de l'article 1 de la loi.

À travers les notions d'incrimination indirecte et de glorification du terrorisme, The Terrorism Bill donne au pouvoir exécutif et aux tribunaux britanniques le pouvoir, non seulement de criminaliser toute forme de soutien à un mouvement social, à une action destinée à faire pression sur le gouvernement anglais, mais aussi de déterminer ce qui est bon et ce qui mauvais partout dans le monde. Cette loi nie l'essence même du politique. Il n'y a plus de conflits d'intérêts, mais une lutte mondiale du bien contre le mal.

Il instaure également une solidarité entre pouvoirs constitués vis-à-vis de leur opposition politique, en criminalisant tout acte de résistance armée, mais aussi de toute action de solidarité matérielle et de soutien, verbal ou écrit, vis-à-vis, par exemple, de la résistance armée tchétchène ou palestinienne ou vis-à-vis de groupes ou de personnes qui défendent, ou qui ont défendu dans le passé de telles luttes. Dans les faits, se positionner politiquement, en désaccord avec le gouvernement britannique, peut devenir un délit.

The Terrorism Bill représente le point le plus avancé dans la mise en place d'un nouvel ordre juridique. Le droit n'est plus ce qui délimite les prérogatives du pouvoir, mais au contraire ce qui supprime toute frontière à son action. L'ordre juridique devient la symbolisation du non-droit. Il s'agit également d'estomper toute délimitation entre la matérialité du fait et sa simple possibilité, le délit existe car le pouvoir en a posé l'éventualité. 